\title{
The superficial temporal artery trunk as a donor vessel in cerebral revascularization: benefits and pitfalls
}

\author{
Ali Alaraj, M.D., William W. Ashley JR., M.D., Ph.D., Fady T. Charbel, M.D., \\ and SEPIDEh Amin-Hanjani, M.D. \\ Department of Neurosurgery, University of Illinois at Chicago, Illinois
}

\begin{abstract}
Object. The superficial temporal artery (STA) is the mainstay of donor vessels for extracranial-intracranial bypass in cerebral revascularization. However, the typically used STA anterior or posterior branch is not always adequate in its flow-carrying capacity. In this report the authors describe the use of the STA trunk at the level of the zygoma as an alternative donor and highlight the benefits and pitfalls of this revascularization option.

Methods. The authors reviewed the cases of 4 patients in whom the STA trunk was used as a donor site for anastomosis of a short interposition vein graft. The graft was implanted into the middle cerebral artery to trap a cartoid aneurysm in 2 patients, and the posterior cerebral artery for vertebrobasilar insufficiency in the other 2 . Discrepancies in size between the interposition vein and STA trunk were compensated for by a beveled end-to-end anastomosis or by implanting the STA trunk into the vein graft in an end-to-side fashion.

Results. Intraoperative flow measurements confirmed the significantly higher flow-carrying capacity of the STA trunk (54-100 ml/minute) compared with its branches $(10-28 \mathrm{ml} / \mathrm{minute})$. The STA trunk interposition graft has several advantages compared with an interposition graft to the cervical carotid, including a shorter graft and no need for a neck incision. However, in the setting of ruptured aneurysm trapping, with the risk of subsequent vasospasm, it is a poor conduit for endovascular therapies.

Conclusions. The STA trunk is a valuable donor option for cerebral revascularization, but should be avoided in the setting of subarachnoid hemorrhage. (DOI: 10.3171/FOC/2008/24/2/E7)
\end{abstract}

\section{KEY WORDS - blood flow - bypass surgery - cerebral revascularization • superficial temporal artery}

$\mathrm{T}$ HE STA-MCA bypass is a technique that provides a conduit for blood flow from the external carotid artery to the MCA territory. This technique was first described by Dr. Yaşargil in $1977^{18}$ and traditionally utilizes either the anterior or posterior branch of the distal STA as an in situ arterial graft. The standard STA-MCA bypass is the workhorse of cerebral revascularization and has been used in the management of cerebovascular occlusive diseases and complex aneurysms. ${ }^{1,3,13,14,18}$ However, the distal STA is not always an adequate source of flow, especially for hemispheric revascularization, necessitating the use of long interposition vein or arterial grafts, typically to the cervical carotid artery. In the present study we provide an update on an alternative strategy: the use of the STA trunk as a donor vessel, with a short vein interposition graft, in 4 cases. The benefits and pitfalls of this alternative revascularization strategy are illustrated and discussed.

Abbreviations used in this paper: $\mathrm{ACA}=$ anterior cerebral artery; ICA = internal carotid artery; MCA = middle cerebral artery; PCA = posterior cerebral artery; SAH = subarachnoid hemorrhage; STA = superficial temporal artery.

\section{Operative Technique}

The general technique for STA dissection has been described previously. ${ }^{5,12}$ Whenever possible a single skin incision incorporating the STA origin and the trajectory of the posterior branch of the STA is made; if this is not possible then the anterior branch is isolated from beneath the skin flap. An 8-10-cm length of the STA is dissected with its surrounding cuff of tissue and wrapped in a papaverinesoaked cottonoid during the craniotomy and exposure. Cut flow measurement in the STA branch is then performed at this stage. ${ }^{1}$ This entails measurement of the flow in the STA using a quantitative ultrasonic flow probe (Charbel MicroFlowprobe; Transonics Systems, Inc.) $)^{2,4}$ after the artery has been transected distally and allowed to bleed freely (Fig. 1). This flow measurement reflects the maximum carrying capacity of the vessel. When the flow in the cut segment is inadequate for the purpose required, or the length of the STA segment is too short to reach the recipient vessel (as in a PCA $\mathrm{P}_{2}$ bypass), then the STA can be truncated down to its stump 1-2 $\mathrm{cm}$ beyond the zygoma, and the cut flow within the STA trunk itself measured. Typically a significantly higher flow will be demonstrated (Table 1). 
If the flow in the trunk is deemed adequate, a short interposition vein graft is used. After the distal anastomosis to the intracranial recipient vessel, the proximal anastomosis is then created to the stump of the STA trunk. A discrepancy in size between the vein (generally 3-4 mm in diameter) and the STA trunk (generally 1.5-2.5 mm) can be compensated in either of 2 ways: 1) the STA can be fishmouthed widely to perform a beveled end-to-end anastomosis (Fig. 2 left) or 2) the STA can be fishmouthed and implanted end-to-side into the proximal aspect of the ligated vein graft (Fig. 2 right). The proximal anastomosis can be performed in a leisurely manner because there is no temporary cerebral ischemia, as may be associated with using a graft from the cervical carotid artery.

\section{Case Reports}

\section{Case 1}

This 58-year-old man presented with symptoms of vertebrobasilar insufficiency. Magnetic resonance imaging of the brain demonstrated posterior fossa infarcts. The patient received a diagnosis of severe intracranial-dominant left vertebral artery stenosis. In anticipation of angioplasty and stenting, the patient was started on aspirin and Plavix, but a significant hemorrhagic conversion of a cerebellar stroke developed, prompting discontinuation of the antiplatelet therapies. This precipitated occlusion of the intracranial stenosis, with subsequent fluctuating symptoms dependent on blood pressure. He was therefore taken to the operating room for posterior circulation revascularization with a PCA bypass. The distal STA branch was too short to reach the $\mathrm{P}_{2}$ segment, so the STA was cut proximally above the zygoma and demonstrated a cut flow rate of $54 \mathrm{ml} / \mathrm{minute}$. An interposition vein graft was performed between the STA and PCA ( $\mathrm{P}_{2}$ segment). Final bypass flow rate was $45 \mathrm{ml} /$ minute. The patient was discharged to an acute rehabilitation facility, and was doing well with a palpable bypass at 3 months.

\section{Case 2}

This 42-year-old man with a prior history of hypertension and smoking presented to an outside institution with crescendo transient ischemic attacks that progressed to slurred speech, lethargy, and obtundation, at which time he was transferred to our institution. Magnetic resonance imaging and angiography of the brain demonstrated an occluded basilar artery, with minimal retrograde flow into the basilar tip. Angiography demonstrated a fetal right PCA origin and no evident angiographic filling into the top of the

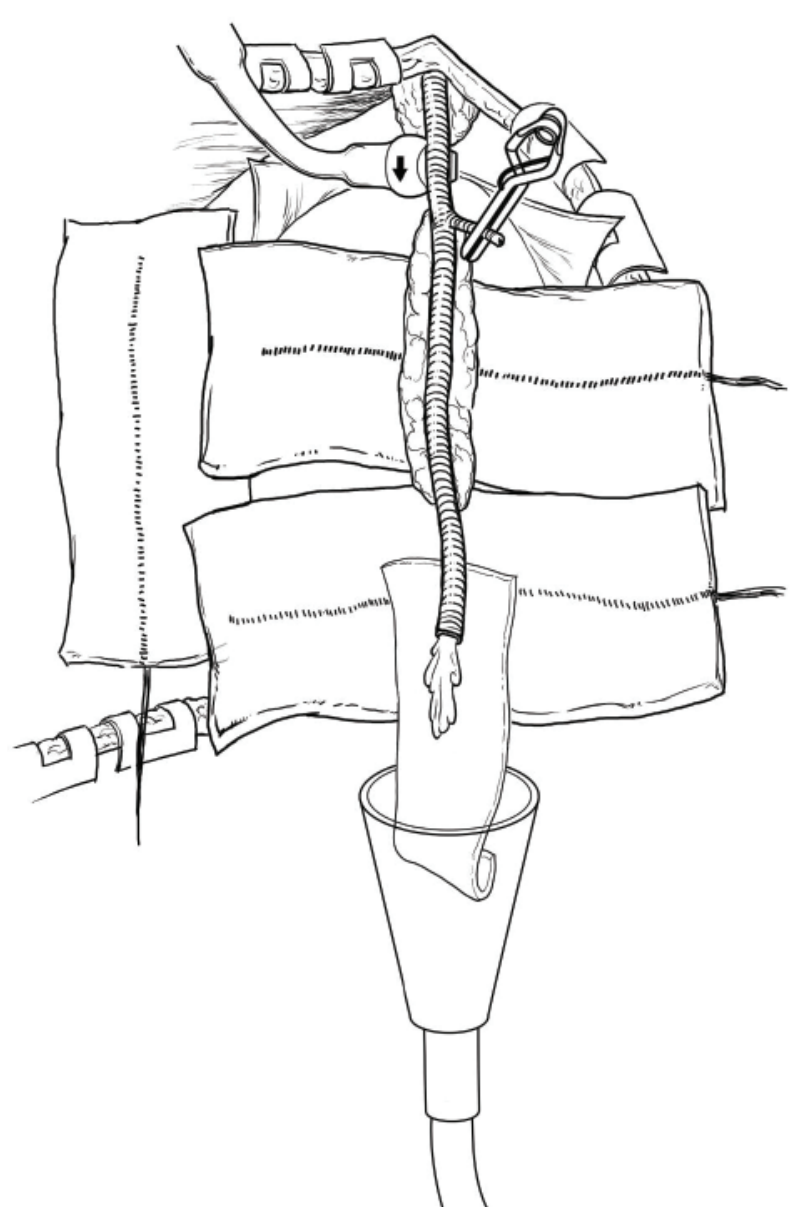

FIG. 1. Illustration of measuring the cut flow: the superficial temporal artery is dissected, the distal end cut open, and the blood flow is measured using the Transonic Flowprobe.

basilar. As a final resort, the patient underwent surgery for posterior circulation flow augmentation. The STA was dissected for $\sim 10 \mathrm{~cm}$; the STA cut flow rate was $10 \mathrm{ml} / \mathrm{minute}$ and was deemed inadequate for flow augmentation. The STA was truncated $2 \mathrm{~cm}$ above the zygoma, and the rate improved to $60 \mathrm{ml} /$ minute. An STA-PCA bypass using an interposition vein graft was constructed. The flow rate in the bypass graft segment after anastomosis was 60 $\mathrm{ml} /$ minute. The graft was patent on angiography 3 weeks postoperatively. However, unfortunately, the patient died of systemic sepsis from pneumonia 1 month later.

TABLE 1

Summary of intraoperative flow measurements of the distal stump and proximal stump as well as the final bypass flow after the bypass has been constructed

\begin{tabular}{cllcrc}
\hline \hline Case No. & \multicolumn{1}{c}{ Diagnosis } & Bypass Type & $\begin{array}{c}\text { Distal STA } \\
\text { Cut Flow }\end{array}$ & $\begin{array}{c}\text { Proximal STA } \\
\text { Cut Flow }\end{array}$ \\
\hline $1^{*}$ & ischemia & STA-PCA & - & $54 \mathrm{ml} / \mathrm{min}$ & $45 \mathrm{ml} / \mathrm{min}$ \\
2 & basilar occlusion & STA-PCA & $10 \mathrm{ml} / \mathrm{min}$ & $60 \mathrm{ml} / \mathrm{min}$ & $60 \mathrm{ml} / \mathrm{min}$ \\
3 & ICA aneurysm & STA-MCA & $28 \mathrm{ml} / \mathrm{min}$ & $100 \mathrm{ml} / \mathrm{min}$ & $85 \mathrm{ml} / \mathrm{min}$ \\
4 & ICA aneurysm & STA-MCA & $10 \mathrm{ml} / \mathrm{min}$ & $89 \mathrm{ml} / \mathrm{min}$ & $95 \mathrm{ml} / \mathrm{min}$ \\
\hline
\end{tabular}

* The distal STA flow was not measured in this patient because it was a short segment. 


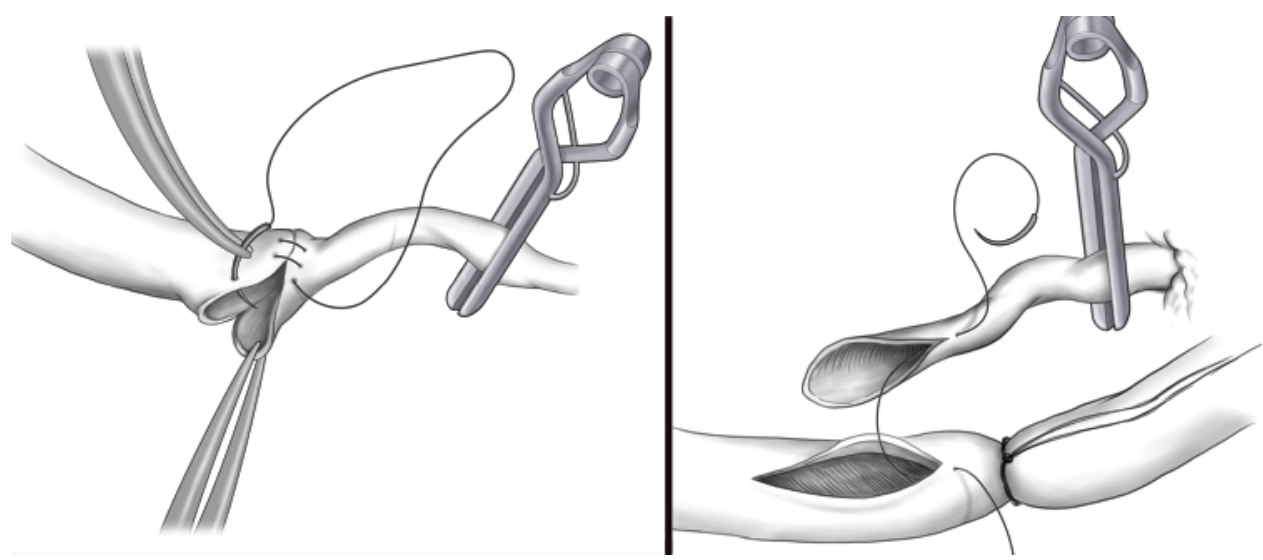

FIG. 2. Left: Illustration of direct end-to-end STA trunk to interposition vein graft. The STA is beveled and fishmouthed to accommodate the discrepancy in the diameter of the 2 vessels. Right: Illustration of end-to-side proximal STA to interposition vein graft. A linear incision is made on the venous side of similar size to the fishmouthed STA. The vein is ligated proximally before anastomosis is performed.

\section{Case 3}

This 44-year-old woman was admitted with a 1-week history of severe headache. A head computed tomography scan showed focal hyperdensity over the right carotid terminus region. Further workup with cerebral angiography showed a dissecting pseudoaneurysm involving the right supraclinoid ICA, which extended into the origin of the ACA. The patient underwent surgery for trapping of the aneurysm with a bypass to the MCA. At the time of surgery, there was fusiform expansion and discoloration of the ICA, but the aneurysm was unruptured. Flow rates were measured intraoperatively in the $\mathrm{M}_{2}$ branches, the sum of which was $81 \mathrm{ml} /$ minute. The STA was dissected and the cut flow rate was measured to be only $28 \mathrm{ml} /$ minute. Therefore the STA branch was felt to be inadequate for revascularization, and was cut at its proximal stump; repeated cut flow measurement through the STA trunk demonstrated 100 $\mathrm{ml} /$ minute. This was considered to be sufficient to replace the MCA. An autologous saphenous vein graft was used as an interposition graft, and an STA trunk-vein- $\mathrm{M}_{2}$ bypass was performed. Blood flow in the bypass following anastomosis and trapping measured $85 \mathrm{ml} /$ minute, almost identical to the recipient bed. The patient did well neurologically and has a patent bypass on angiography 6 months postoperatively.
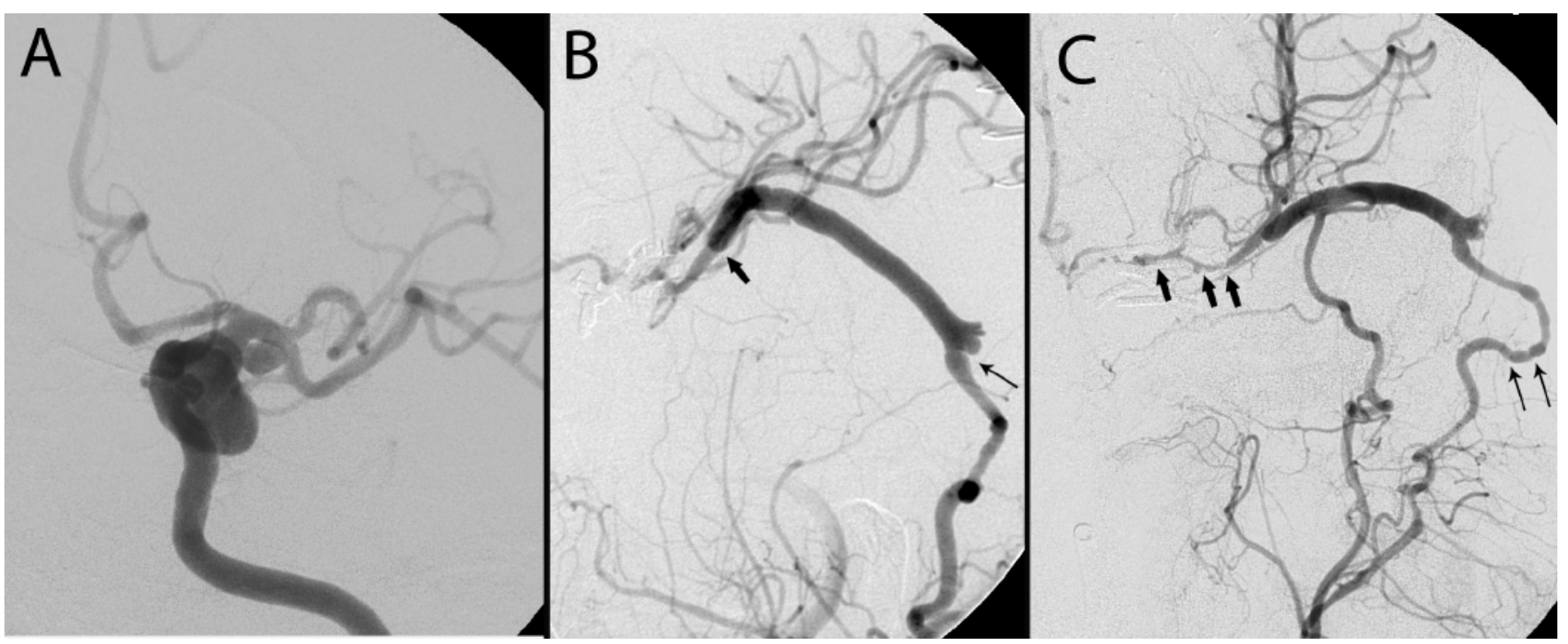

FIG. 3. Case 4 A. Initial angiogram showing the large left posterior communicating artery region aneurysm, with a smaller anterior choroidal artery aneurysm B. Day 5 postoperative angiogram showing the patent STA-vein-MCA bypass. The end-to-side anastomosis (long arrow) between the STA and the vein is seen. The distal anastomosis between the vein graft and the MCA (short arrows) is visualized. The aneurysm is trapped. C. Day 7 postoperative angiogram showing the presence of vasospasm involving the upper cervical STA, proximal to the anastomosis (long arrows), and diffuse severe vasospasm involving the $\mathrm{MCA}\left(\mathrm{M}_{1}\right.$ and $\mathrm{M}_{2}$ segments) (short arrows). 


\section{Case 4}

This 50-year-old woman presented with a Grade III SAH. Her initial angiogram demonstrated a ruptured 15mm-long left posterior communicating artery region aneurysm with a dysplastic fusiform ICA dilation, in addition to a smaller 5-mm left anterior choroidal artery aneurysm (Fig. 3A). The patient underwent craniotomy for surgical trapping of the fusiform ICA segment and aneurysm. During temporary ICA occlusion distal to the aneurysm, minimal collateral flow was present across the anterior communicating artery, based on intraoperative flow measurement of the $\mathrm{M}_{1}$ branch (fall from $70 \mathrm{ml} /$ minute to $5 \mathrm{ml} /$ minute with temporary clipping). The STA branch was dissected free for $8 \mathrm{~cm}$, and the cut flow rate was only $10 \mathrm{ml} /$ minute. The STA was cut proximally, and a repeated flow measurement in the proximal STA stump was $89 \mathrm{ml} / \mathrm{minute}$. An interposition venous graft was used between the proximal STA trunk and the $\mathrm{M}_{2}$ segment. Flow measurement in the bypass was $95 \mathrm{ml} / \mathrm{minute}$ after aneurysm trapping, and Day 5 postoperative angiography confirmed good bypass filling and obliteration of the aneurysms (Fig. 3B). The patient initially recovered well, but on postoperative Day 7 (Fig. 3C), right hemiparesis and speech difficulty developed due to a severe intracranial vasospasm. The patient underwent multiple cerebral angiograms and intraarterial verapamil infusions through the bypass. The endovascular therapy was limited by the easy and severe spasmogenicity of the STA in response to cannulation for intraarterial injections. Furthermore, access for angioplasty could not be obtained due to the size and spasmogenicity of the proximal STA. The patient suffered patchy areas of stroke in the left hemisphere. She was discharged to a rehabilitation facility. The bypass was palpable at her 3-month follow-up office visit.

\section{Discussion}

Cerebral revascularization using a short interposition vein graft from the STA trunk has been reported in the past. ${ }^{7-9,11,16,17}$ In 1983 Little et al. ${ }^{11}$ reported the largest experience published to date, with 19 bypass procedures using the STA trunk as a donor vessel; their early patency rate was $90 \%$. The authors proposed that this procedure might be useful as a primary means of cerebral revascularization or as an alternative approach when the distal STA branch cannot be used because of its small size, the severity of arteriosclerotic changes, or damage during its dissection. The use of the STA trunk was also described by Spetzler and colleagues ${ }^{16}$ in creating the "bonnet" bypass. This technique entails the use of the contralateral STA trunk as a donor for an interposition radial artery graft to the MCA in cases of skull base tumors with limited access to ipsilateral donor vessels. ${ }^{7,16}$ The use of the STA trunk has been described for trapping and revascularization of a giant ACA aneurysm, with STA- $\mathrm{A}_{3}$ arterial interposition graft and subsequent $\mathrm{A}_{3}-\mathrm{A}_{3}$ side-to-side bypass. ${ }^{8}$ Cases of saphenous vein or radial artery interposition grafting between the donor STA trunk and recipient ACA have also been reported for revascularization in cerebrovascular occlusive disease. ${ }^{9,17}$

The premise underlying the use of the STA trunk relates to Poiseuille's law and flow dynamics, the physical law concerning the laminar flow [Q] of an incompressible uniform viscous liquid through a cylindrical tube. As such, the law is applicable to the corporeal circulation. The equation relevant to Poiseuille's law is as follows: $\mathrm{Q}=\left(\pi \mathrm{r}^{4} \mathrm{P}\right) /(8 \eta \mathrm{L})$ Where $\mathrm{r}$ represents the internal radius of the blood vessel, $P$ the pressure at the vessel end, $\eta$ the dynamic blood viscosity, and $\mathrm{L}$ the total length of the tube in that direction. Poiseuille's law relates the flow rate to the pressure, viscosity, vessel radius, and length. Because flow is related to the radius raised to the fourth power, a minimal change in the radius of a vessel will have an exponential impact on the rate of blood flow. Additionally the length of the vessel conduit will impact blood flow inversely. Compared to the typical STA branch, the STA trunk is significantly shorter and generally of larger diameter than the distal branch. Based on flow dynamics principles, therefore, the STA trunk would be expected to have a significantly higher flow capacity than its branches. This principle is well demonstrated in our cases, in which intraoperative cut flow measurements showed an increase in flows from the 10-28 $\mathrm{ml} / \mathrm{minute}$ range to the $60-100 \mathrm{ml} / \mathrm{minute}$ range in the 3 cases in which both the STA branch and trunk were measured.

Flow rates in the STA branch can vary widely ${ }^{1}$ as a result of a combination of factors including not only diameter and length, but also the presence of arteriosclerosis within the STA segment itself. The STA is prone to progressive intimal thickening and alteration of the elastic lamina. ${ }^{10}$ The presence of atherosclerosis within the STA branch or trunk can lead to decrease in the flow within that segment, thus rendering it unsuitable for use. Therefore, direct intraoperative measurement of the cut flow rate is an important step in determining the actual flow-carrying capacity of the STA and establishing adequacy of the donor vessel.

There are several advantages to use of the STA trunk as a donor compared to the cervical carotid artery (external or common). Use of the STA trunk allows the use of a short interposition graft (approximately $6-8 \mathrm{~cm}$ ) compared to the significantly longer graft $(18-20 \mathrm{~cm})$ required for a vein or arterial graft in the cervical region. It is well-accepted that a shorter interposition graft is desirable for graft longevity and patency. ${ }^{6,15}$ The use of the STA trunk can avoid the need for a neck incision and dissection, and also reduce the size of the incision and the length of time for harvesting of the shorter vein or arterial graft. In addition, occlusion of the STA trunk for the purpose of anastomosis poses no risk of cerebral ischemia, whereas cases requiring bypass to the common carotid rather than the external carotid artery (such as in a high bifurcation) would have the potential for ischemia during temporary clipping.

There are pitfalls to use of the STA trunk, however. This is well-illustrated in 1 of our cases (Case 4), in which the STA trunk with an interposition vein graft was adequate to replace the flow at the time of aneurysm trapping. However, once an intracranial vasospasm developed in the patient as a consequence of SAH, the choice of donor vessel limited the extent and efficacy of endovascular interventions. The STA is too small to provide a conduit for a microcatheter for balloon angioplasty, and even cannulation for injection of intraarterial vasorelaxants can induce vasospasm easily in the STA itself, exacerbating cerebral ischemia by reducing flow in the bypass. Consequently, for ruptured aneurysms, a large-caliber interposition vein graft 


\section{Superficial temporal artery trunk in cerebral revascularization}

from the cervical carotid artery (external or common) would be a more appropriate choice; the vein can be cannulated with microcatheters for intraarterial injections and used as a conduit for balloon angioplasty, as needed if vasospasm develops.

\section{Conclusions}

The STA trunk is a valuable donor option to consider for use in surgical cerebral revascularization, but should be avoided in the setting of SAH.

\section{Financial Disclosure}

Dr. Fady T. Charbel is a consultant for Transonics Systems, Inc.

\section{References}

1. Amin-Hanjani S, Du X, Mlinarevich N, Meglio G, Zhao M, Charbel FT: The cut flow index: an intraoperative predictor of the success of extracranial-intracranial bypass for occlusive cerebrovascular disease. Neurosurgery 56:75-85, 2005

2. Amin-Hanjani S, Meglio G, Gatto R, Bauer A, Charbel FT: The utility of intraoperative blood flow measurement during aneurysm surgery using an ultrasonic perivascular flow probe. Neurosurgery 58: ONS305-ONS312, 2006

3. Anonymous: Failure of extracranial-intracranial arterial bypass to reduce the risk of ischemic stroke. Results of an international randomized trial. The EC/IC Bypass Study Group. N Engl J Med 313:1191-1200, 1985

4. Charbel FT, Hoffman WE, Misra M, Ostergren L: Ultrasonic perivascular flow probe: technique and application in neurosurgery. Neurol Res 20:439-442, 1998

5. Charbel FT, Meglio G, Amin-Hanjani S: Superficial temporal artery-to-middle cerebral artery bypass. Neurosurgery 56 (1 Suppl):186-190, 2005

6. Couldwell WT, Zuback J, Onios E, Ahluwalia BS, Tenner M, Moscatello A: Giant petrous carotid aneurysm treated by submandibular carotid-saphenous vein bypass. Case report. J Neurosurg 94:806-810, 2001
7. Deshmukh VR, Porter RW, Spetzler RF: Use of "bonnet" bypass with radial artery interposition graft in a patient with recurrent cranial base carcinoma: technical report of two cases and review of the literature. Neurosurgery 56 (1 Suppl): E202, 2005

8. Inoue T, Tsutsumi K, Ohno H, Shinozaki M: Revascularization of the anterior cerebral artery with an A3-a3 anastomosis and a superficial temporal artery bypass using an A3-radial artery graft to trap a giant anterior communicating artery aneurysm: technical case report. Neurosurgery 57 (1 Suppl): E207, 2005

9. Iwata Y, Mizuta T, Takemoto O, Shimizu K, Nakatani S: An interposed superficial temporal artery graft bypass for anterior cerebral artery ischemia. Microsurgery 9:14-17, 1988

10. Lie JT, Brown AL Jr, Carter ET: Spectrum of aging changes in temporal arteries. Its significance, in interpretation of biopsy of temporal artery. Arch Pathol 90:278-285, 1970

11. Little JR, Furlan AJ, Bryerton B: Short vein grafts for cerebral revascularization. J Neurosurg 59:384-388, 1983

12. Newell DW, Vilela MD: Superficial temporal artery to middle cerebral artery bypass. Neurosurgery 54:1441-1449, 2004

13. Peerless SJ, Ferguson GG, Drake CG: Extracranial-intracranial (EC/IC) bypass in the treatment of giant intracranial aneurysms. Neurosurg Rev 5:77-81, 1982

14. Sekhar LN, Kalavakonda C: Cerebral revascularization for aneurysms and tumors. Neurosurgery 50:321-331, 2002

15. Sekhar LN, Sen CN, Jho HD: Saphenous vein graft bypass of the cavernous internal carotid artery. J Neurosurg 72:35-41, 1990

16. Spetzler RF, Roski RA, Rhodes RS, Modic MT: The "bonnet bypass". Case report. J Neurosurg 53:707-709, 1980

17. Tanaka K, Yonekawa Y, Satou K, Katagiri K, Kouno H: [STAACA anastomosis with interposed vein graft; a case report.] No Shinkei Geka 20:171-176, 1992 (Jpn)

18. Yaşargil MG, Yonekawa Y: Results of microsurgical extra-intracranial arterial bypass in the treatment of cerebral ischemia. Neurosurgery 1:22-24, 1977

Manuscript submitted November 15, 2007.

Accepted December 10, 2007.

Address correspondence to: Sepideh Amin-Hanjani, M.D., Neuropsychiatric Institute (MC 799), Department of Neurosurgery, University of Illinois at Chicago, 912 South Wood Street, Chicago, Illinois 60612-5970. email: hanjani@uic.edu. 